\title{
Eprex warning issued, but no ban
}

Drug regulators in Australia and Europe have banned the subcutaneous injection of the antianemia drug, epoetin alfa (Eprex) due to its association with pure red cell aplasia (PRCA), but Health Canada despite issuing 3 advisories about the Janssen-Ortho drug (CMAF $2002 ; 166[4]: 480)$ - has yet to follow suit.

On Jan. 20, Health Canada advised against intravenous injection for patients with chronic renal failure (CRF). If IV access is unavailable, it recommended a risk-benefit assessment prior to subcutaneous administration.

"Eprex is not banned because the benefits continue to outweigh the risks when the product is used according to the

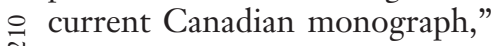
ờ said Health Canada spokesperson Jirina Vik.

The Canadian Society of Nephrology advises its members that epoetin alfa "be administered via the IV route whenever ๑ feasible.”
As of January 2003, the drug was linked to 179 cases or suspected cases of PRCA outside the US. Health Canada reports about 80. Most have involved CRF patients treated subcutaneously with Eprex polysorbate-80 (HSA-3) in prefilled syringes. A decreased frequency of reports in 2003 (only 5 reports worldwide as of Aug. 31) coincides with an increase in use of the vial version (i.e., IV injected) of the drug, says Janssen-Ortho spokesperson Simone Philogene.

The link to PRCA, which results in a life-long dependence on blood transfusions, appears to be limited to the Eprex formulary manufactured by Janssen-Ortho or its parent, Johnson \& Johnson (J\&J). In the US, where epoetin alfa is manufactured by Amgen, the number of PRCA-associated cases are 50-fold lower.

Epoetin alfa is prescribed to people who are suffering from anemia, HIV, cancer or kidney failure.
Rates of PRCA rose after 1998 when prefilled syringes became available and subcutaneous use increased. At the same time, J\&J removed a humanderived stabilizing serum in the wake of concerns about Creutzfeldt-Jakob disease, and began using a synthetic stabilizer. J\&J scientists say the new formula is more prone to damage from improper handling; exposure to heat or excessive shaking might cause the proteins to clump, making them more likely to stimulate an immune response. They also suspect that subcutaneous injection could magnify any immune reaction.

In December 2002, health regulators in Australia and Europe banned subcutaneous use of the drug for CRF patients. Sales of epoetin alfa, which totalled $\$ 13$ billion worldwide in 2001 , fell $5 \%$ in the fourth quarter of 2002.

An alternate product, Arnasep (by Amgen) was approved for use in Canada in 2002. - Barbara Sibbald, CMAJ 